\title{
Breast Cancer Screening Practice: Awareness among Women in Muar District
}

Roy Rillera Marzo, Amaluddin Ahmad, Turani Talukder, Myat Thida Win, Maung Maung Soe

Asia Metropolitan University

\begin{abstract}
Introduction: Breast cancer is the most common cancer among women. The most recent estimate indicated that there are more than 1.6 million new cases of breast cancer worldwide yearly.
\end{abstract}

\section{Objectives:}

To assess the level of awareness on breast cancer screening practice among women in Muar.

Methods: This is a quantitative cross-sectional survey done on 383 respondents in Muar where participants' age ranged from 18-80 years. The study used a two-step sampling method namely cluster and simple random sampling. Descriptive analysis was used to describe participants' socio-demographic characteristics while chi square was used to present the results.

Results: Conventional method of breast cancer awareness campaign currently available is probably unsatisfactory. In our survey, only $52.38 \%$ of vulnerable aged women among our study subjects in Muar have any knowledge or awareness on breast cancer screening practices. Factors influencing knowledge and awareness of breast cancer screening practices were greatly influenced by level of education and level of household monthly income.

Only 50 to $60 \%$ of respondents in group 1 (18-25 years), group 2 (26-31 years), group 3 (3237 years) performs Breast Self-Examination (BSE). The practice of clinical breast examination among women in group 4 (38-43 years) group 5 (44-50 years) and group 6 (>50 years) only ranged between as low as $30 \%$ to a maximum of $50 \%$ while annual mammogram screening was only $20 \%$ in group 5 and $30 \%$ in group 6 .

Conclusion: The results of the study proved poor knowledge and practices of breast screening practices among vulnerable women in Muar district. Compliance to clinical breast examination and mammogram is even lower. 